BCCUNY-HEP 2004/01

February 2, 2004

Revised, February 24,2004

\title{
GAUGE-INVARIANT COORDINATES ON GAUGE-THEORY ORBIT SPACE
}

\author{
Peter Orland $d^{\text {a.b.1 }}$ \\ a. Physics Program, The Graduate School and University Center, The City University \\ of New York, 365 Fifth Avenue, New York, NY 10016, U.S.A. \\ b. Department of Natural Sciences, Baruch College, The City University of New York, \\ 17 Lexington Avenue, New York, NY 10010, U.S.A., orland@gursey.baruch.cuny.edu
}

\begin{abstract}
A gauge-invariant field is found which describes physical configurations, i.e. gauge orbits, of non-Abelian gauge theories. This is accomplished with non-Abelian generalizations of the Poincaré-Hodge decomposition formula for one-forms. In a particular sense, the new field is dual to the gauge field. Using this field as a coordinate, the metric and intrinsic curvature are discussed for Yang-Mills orbit space for the $(2+1)$ and (3+1)-dimensional cases. The sectional, Ricci and scalar curvatures are all formally non-negative. An expression for the new field in terms of the Yang-Mills connection is found in $2+1$ dimensions. The measure on Schrödinger wave functionals is found in both $2+1$ and $3+1$ dimensions; in the former case, it resembles Karabali, Kim and Nair's measure. We briefly discuss the form of the Hamiltonian in terms of the dual field and comment on how this is relevant to the mass gap for both the $(2+1)$ - and (3+1)-dimensional cases.
\end{abstract}

\footnotetext{
${ }^{1}$ Work supported in part by a PSC-CUNY Research Award
} 


\section{Introduction}

Many field theorists have speculated as to whether QCD can be solved by the substitution of the Yang-Mills connection by gauge-invariant degrees of freedom. The source of this speculation is that the space of physical degrees of freedom or gauge orbits $\mathcal{M}$ is the quotient of connection space $\mathbb{A}$ by gauge transformations $\mathcal{G}$, neatly written as

$$
\mathcal{M}=\mathbb{A} / \mathcal{G} \text {. }
$$

A gauge orbit $\mathrm{O}$ is an equivalence class containing all gauge transforms of some gauge field $A_{k}^{g}=g^{-1} A_{k} g^{-1}+\mathrm{i} g^{-1} \partial_{k} g$. Schrödinger wave functionals depend on orbits - not gauge fields. Reformulating Yang-Mills theory in terms of gauge orbits can be accomplished in principle (except on a set of measure zero) by gauge fixing and eliminating Gribov copies [1, 2, 3, 4, 5, 6]. The quest for a gauge-invariant formalism of gauge theories began decades ago [7. A radical idea is to find a string-model reformulation; remarkably, such a reformulation been quite successful for non-asymptotically-free gauge theories [8].

In this paper, we find a relatively simple way of characterizing coordinate charts of $\mathcal{M}$ as hypersurfaces in $\mathbb{A}$. These hypersurfaces are cross sections of the fiber bundle, and there are Gribov copies. Many of the geometric properties of orbit space are much easier to understand, however, than with ordinary gauge-fixing procedures. For example, the metric tensor and intrinsic curvature are simple to obtain. A single-valued definition of the coordinates exists, at least in $2+1$ dimensions, which solves the Gribov problem. In $2+1$ dimensions, there appears to be a connection with the remarkable methods of Karabali and Nair [9] and of Karabali, Kim and Nair [10].

We work only with $\mathrm{SU}(N)$ gauge fields in Hamiltonian formalism in $D$ space and one time dimension. The gauge connection $D_{j}=\partial_{j}-\mathrm{i} A_{j}$ where $j=1, \ldots, D$ and $A(x)_{j}=\sum_{\alpha} A(x)_{j}^{\alpha} t_{\alpha}$ is a traceless, Hermitian $N \times N$ matrix field. The basis of the Lie algebra consists of generators $t^{\alpha}$, with $\left[t_{\alpha}, t_{\beta}\right]=i \sum_{\gamma} f_{\alpha \beta}^{\gamma} t_{\gamma}$. For any $N \times N$ matrix field $Q$, we define $\mathcal{D}_{j} Q=\left[D_{j}, Q\right]$ and the gauge field in the adjoint representation by $\mathcal{A}_{j}$ by $\mathcal{D}_{j}=\partial_{j}-\mathrm{i} \mathcal{A}_{j}$. The field strengths in the fundamental representation and the adjoint representation are $F_{j k}=\mathrm{i}\left[D_{j}, D_{k}\right]$, and $\mathcal{F}_{j k}=\mathrm{i}\left[\mathcal{D}_{j}, \mathcal{D}_{k}\right]$, respectively.

We adopt a notation suited to the discussion of components of vectors and tensors on connection space and orbit space. We will write the gauge field as

$$
A^{(x, j, \alpha)}=A(x)_{j}^{\alpha},
$$

and Lie-Algebra-valued scalar fields similarly, e.g.

$$
\Phi^{(x, \alpha)}=\Phi(x)^{\alpha} .
$$

Let us define A to consist of square-integrable connections on infinite space (not space-time) $\mathbb{R}^{\mathrm{D}}$, with square-integrable field strengths $F$.

The metric on orbit space $\mathcal{M}$, is

$$
d \rho^{2}=\int G_{(x, j, \alpha)(y, k, \beta)} \delta A^{(x, j, \alpha)} \delta A^{(y, k, \beta)},
$$


where the integration denotes a sum over all repeated indices (including $x$ and $y$ !) and the metric is

$$
G_{(x, j, \alpha)(y, k, \beta)}=\left[\delta_{j k} \delta^{\alpha \beta}-\mathcal{D}_{j}\left(P \frac{1}{\mathcal{D}^{2}}\right) \mathcal{D}_{k}\right] \delta^{\mathrm{D}}(x-y)
$$

where $P$ denotes the principle value. This metric is the projection which removes those variations of the gauge field of the form

$$
\delta A^{(x, j, \alpha)}=\mathcal{D}_{\beta}^{\alpha} \Omega^{((x, \beta))} .
$$

The reason for the double-bracket notation $((x, \beta))$ is to distinguish variations which are gauge transformations from those which are not.

The metric (1.1) was argued by Babelon and Viallet [3] to be the infinitesimal version of the distance function between an orbit $\mathrm{O}$ containing gauge field $A$ and an orbit $\mathrm{O}^{\prime}$ containing gauge field $A^{\prime}$

$$
\rho\left(\mathrm{O}, \mathrm{O}^{\prime}\right)^{2}=\frac{1}{2} \inf _{g \in \mathcal{G}} \int d^{\mathrm{D}} x\left\{\left[A^{g}(x)\right]^{2}-A^{\prime}(x)^{2}\right\} .
$$

This was proven for appropriately defined gauge fields as Hilbert-space vectors in reference 4. There it was also shown by a simple argument that the size of orbit space is unbounded in $3+1$ dimensions - the potential energy $\int \operatorname{Tr} F_{i j}^{2}$ can even be arbitrarily small at sufficiently large distances from a pure gauge. This indicates that proofs that orbit space is bounded [11] do not apply to the thermodynamic limit. Feynman attempted to explain confinement and the mass gap in $2+1$ dimensions by approximating this distance on configurations of small magnetic energy [12]. The object of Feynman's investigation was to show that such configurations lie within a finite diameter. Orland and Semenoff showed that Feynman's distance approximations were incorrect, but were actually able to calculate some distances exactly [6], supporting this idea.

The virtue of the expression on the right-hand side of (1.2), namely that is has gauge transformations as zero modes (1.3), is also its curse. Metric tensors with zero eigenvalues are rather difficult to work with. A formalism to deal with the geometry from this viewpoint has been discussed in reference 4]. An alternative, which we believe to be more useful, is presented below.

In the next section we present a non-Abelian decomposition formula for differential one-forms. This provides a powerful new gauge-fixing method in Section 3, and we use this to study the gauge-fixed hypersurface in the space of connections. Gribov singularities are realized as the appearance of harmonic one-forms in the decomposition formula. We elaborate on some issues concerning gauge invariance in Section 4. In Section 5, we find formal expressions for the extrinsic and (intrinsic) curvature tensor of the hypersurface. The Ricci and scalar curvatures are discussed for arbitrary space dimension in Section 6. We briefly examine the Yang-Mills Hamiltonian formulated on the hypersurface in Section 7. In Section 8, we show how to explicitly obtain the hypersurface coordinate in terms of the gauge connection in $2+1$ dimensions; this is 
used to find a Hamiltonian formalism which strongly resembles that of Karabali, Kim and Nair [9, 10] in Section 9. We introduce a dual Hamiltonian and very briefly discuss how the Yang-Mills mass gap arises in Section 10. Our conclusions and directions for further work are summarized in Section 11.

\section{Non-Abelian Poincaré-Hodge decomposition for- mulas}

Let us briefly recall the Poincaré-Hodge decomposition formula. Any differential form $C$ may be decomposed as

$$
C=d \Phi+*[d(* \Omega)]+h
$$

where $h$ is a harmonic form; by which it is meant that $h$ is both closed $d h=0$, and co-closed $d * h=0$. For two dimensions of space, and a a one-form $C=\sum_{j} C^{(x, j)} \cdot d x^{j}$ (2.1) may be written as

$$
C^{(x, j)}=\epsilon^{j k} \partial_{k} \Phi^{(x)}+\partial_{j} \Omega^{(x)}+h^{(x, j)},
$$

and for three space dimensions

$$
C^{(x, j)}=\epsilon^{j k l} \partial_{k} \Phi^{(x, l)}+\partial_{j} \Omega^{(x)}+h^{(x, j)} .
$$

Harmonic forms are particularly important in finite volumes. The dimensions of the space of harmonic forms are the Betti numbers of the manifold. For some special finitevolume manifolds, namely star-shaped regions, there are no harmonic forms, hence from (2.1) any closed form $C, d C=0$ must be exact $C=d \Phi$.

What is especially significant about the decomposition of forms is that it is a decomposition into subspaces of Hilbert space. Consider a second one-form in two space dimensions:

$$
C^{\prime(x, j)}=\epsilon^{j k} \partial_{k} \Phi^{\prime(x)}+\partial_{j} \Omega^{\prime(x)}+h^{\prime(x, j)} .
$$

Then the inner product between $C$ and $C^{\prime}$ decomposes into three pieces:

$$
\left\langle C^{\prime} \mid C\right\rangle=\int C^{\prime(x, j)} C^{(x, j)}=\int \Phi^{\prime(x)}\left(-\partial^{2}\right) \Phi^{(x)}+\int \Omega^{\prime(x)}\left(-\partial^{2}\right) \Omega^{(x)}+\int h^{\prime(x, j)} h^{(x, j)} .
$$

A similar expression holds for the inner product in three space dimensions.

Consider a non-Abelian infinitesimal functional variation $\delta A^{(x, j, \alpha)}$ which is covariantly divergenceless, i.e. $\mathcal{D}^{\gamma}{ }_{\alpha} \delta A^{(x, j, \alpha)}=0$. For our purposes, we must also assume that the Laplacian on scalars $\mathcal{D}^{2}$ has no zero modes. We shall discuss this issue further at the end of Section 4 . We say that $\delta A$ is non-Abelian exact in two space dimensions if

$$
\delta A^{(x, j, \alpha)}=\int L_{(y, \beta)}^{(x, j, \alpha)} \delta \Phi^{(y, \beta)},
$$


where we have introduced the linear mapping

$$
L_{(y, \beta)}^{(x, j, \alpha)}=\frac{\delta A^{(x, j, \alpha)}}{\delta \Phi^{(y, \beta)}}=\left[\epsilon^{j k} \mathcal{D}_{k}+\mathrm{i} \mathcal{D}_{j} \frac{1}{\mathcal{D}^{2}} \mathcal{F}_{12}\right]_{\beta}^{\alpha} \delta^{2}(x-y)
$$

and the integration is over $y$, with summation over $\beta$. The expressions $i \mathcal{D}_{j}$ and $\frac{1}{\mathcal{D}^{2}}$ in (2.3) are to be understood as self-adjoint operators; they act on everything to the right. We do not discuss the domain of $L$, but are confident that it can be determined [14. We say that $\delta A$ is non-Abelian exact in three space dimensions if

$$
\delta A^{(x, j, \alpha)}=L_{(y, l, \beta)}^{(x, j, \alpha)} \delta \Phi^{(y, l, \beta)}
$$

where

$$
L_{(y, l, \beta)}^{(x, j, \alpha)}=\frac{\delta A^{(x, j, \alpha)}}{\delta \Phi^{(y, l, \beta)}}=\left[\epsilon^{j k l} \mathcal{D}_{k}+\mathrm{i} \mathcal{D}_{j} \frac{1}{\mathcal{D}^{2}}(* \mathcal{F})^{(x, l)}\right]_{\beta}^{\alpha} \delta^{3}(x-y)
$$

and the components of the magnetic field are $(* \mathcal{F})^{(x, l)}=\frac{1}{2} \epsilon^{l m n} \mathcal{F}_{m n}$, in the standard notation of Hodge.

A non-Abelian exact variation $\delta A$ in either two or three space dimensions satisfies $\mathcal{D} \cdot \delta A=0$. This can be readily checked for (2.2) and (2.4).

Before going on to the non-Abelian generalization of the decomposition formula, let us take stock of the progress we have made. The expressions (2.2), (2.3), (2.4) and (2.5) are powerful tools for understanding the geometry of orbit space. We can write the metric (1.1) (1.2) for two-space-dimensional orbits as

$$
d \rho^{2}=\int G_{(x, \alpha)(y, \beta)} \delta \Phi^{(x, \alpha)} \delta \Phi^{(y, \beta)},
$$

where

$$
G_{(x, \alpha)(y, \beta)}=\int L_{(x, \alpha)}^{(z, j, \gamma)} \underset{(y, \beta)}{(z, j, \gamma)}=\left(-\mathcal{D}^{2}+\mathcal{F}_{12} \frac{1}{-\mathcal{D}^{2}} \mathcal{F}_{12}\right)_{\alpha \beta} \delta^{2}(x-y),
$$

which is a non-negative quadratic form.

From (2.6) we see that $\delta \Phi^{(x, \alpha)}$ may be interpreted as the functional variation of a scalar field $\Phi^{(x, \alpha)}$. It is this scalar field which is a natural gauge-invariant set of coordinates.

In three dimensions, there is a subtlety, namely that the operator $L_{(y, l, \beta)}^{(x, j, \alpha)}$ has zero modes. The quantity $\delta \Phi^{(x, l, \alpha)}$ may be interpreted as the functional variation of a vector field $\Phi^{(x, l, \alpha)}$. Physically, this field is a new gauge field which is dual to the original gauge field. The zero modes of $L_{(y, l, \beta)}^{(x, j, \alpha)}$ are the dual gauge transformations. These gauge transformations are complicated and will not be discussed in detail here. It is no clear whether any harm results to the physics by fixing or even breaking the dual gauge invariance. For the time being we fix the dual gauge invariance by excluding $l=3$ from $L_{(y, l, \beta)}^{(x, j, \alpha)}$. This is a "dual axial-gauge condition" in which $\Phi^{(x, 3, \alpha)}$ is set to a constant. 
The dual-gauge-fixed vector field $\Phi^{(x, l, \beta)}$ is a coordinate we can use on orbit space in three dimensions.

The metric in three space dimensions is

$$
d \rho^{2}=\int G_{(x, l, \alpha)(y, m, \beta)} \delta \Phi^{(x, l, \alpha)} \delta \Phi^{(y, m, \beta)},
$$

where $l, m=1,2$ and

$$
\begin{gathered}
G_{(x, l, \alpha)(y, m, \beta)}=\int L_{(x, l, \alpha)}^{(z, j, \gamma)} L_{(y, m, \beta)}^{(z, j, \gamma)} \\
=\left[-\mathcal{D}^{2} \delta^{l m}+\mathcal{D}_{m} \mathcal{D}_{l}+(* \mathcal{F})_{l} \frac{1}{-\mathcal{D}^{2}}(* \mathcal{F})_{m}\right]_{\alpha \beta} \delta^{3}(x-y), \\
=\left[-\mathcal{D}^{2} \delta^{l m}+\mathcal{D}_{l} \mathcal{D}_{m}-\mathrm{i} \mathcal{F}_{l m}+(* \mathcal{F})_{l} \frac{1}{-\mathcal{D}^{2}}(* \mathcal{F})_{m}\right]_{\alpha \beta} \delta^{3}(x-y),
\end{gathered}
$$

which is a non-negative symmetric quadratic form.

The non-Abelian decomposition formula in two space dimensions is

$$
\delta A^{(x, j, \alpha)}=\int L_{(y, \beta)}^{(x, j, \alpha)} \delta \Phi^{(y, \beta)}+\int \mathcal{D}_{((z, \sigma))}^{(x, j, \alpha)} \delta \Omega^{((z, \sigma))}+h^{(x, j, \alpha)}
$$

where

$$
\mathcal{D}_{((z, \sigma))}^{(x, j, \alpha)}=\left(\mathcal{D}_{j}\right)^{\alpha}{ }_{\sigma} \delta^{2}(x-z)
$$

and where the vector $h^{(x, j, \alpha)}$ satisfies the non-Abelian "harmonicity" conditions, which we call non-Abelian closed and non-Abelian co-closed, respectively:

$$
\begin{gathered}
\int\left(\mathcal{D}^{\dagger}\right)_{(x, j, \alpha)}^{((z, \sigma))} h^{(x, j, \alpha)}=0, \\
\int\left(L^{\dagger}\right)_{(x, j, \alpha)}^{(y, \beta)} h^{(x, j, \alpha)}=0 .
\end{gathered}
$$

Notice that $\delta \Omega^{((z, \sigma))}$ is a gauge transformation.

If there is another variation $\delta A^{(x, j, \alpha)}$, of $A^{(x, j, \alpha)}$, we may decompose it as

$$
\delta A^{\prime(x, j, \alpha)}=\int L_{(y, \beta)}^{(x, j, \alpha)} \delta \Phi^{\prime(y, \beta)}+\int \mathcal{D}_{((z, \sigma))}^{(x, j, \alpha)} \delta \Omega^{\prime((z, \sigma))}+h^{\prime(x, j, \alpha)}
$$

which yields the decomposition of the inner product: 


$$
\begin{aligned}
\int \delta A^{\prime(x, j, \alpha)} \delta A^{(x, j, \alpha)}=\int G_{(u, \alpha)(v, \beta)} \delta \Phi^{\prime(u, \alpha)} \delta \Phi^{(v, \beta)} & +\int\left(-\mathcal{D}^{2}\right)_{((z, \gamma))((w, \rho))} \delta \Omega^{\prime((z, \gamma))} \delta \Omega^{((w, \rho))} \\
& +\int h^{\prime(x, j, \alpha)} h^{(x, j, \alpha)}
\end{aligned}
$$

Thus $\delta \Phi^{(y, \beta)}$ produces a variation of the gauge field which is orthogonal to gauge transformations.

In three space dimensions, we have the decomposition formula

$$
\delta A^{(x, j, \alpha)}=\int L_{(y, l, \beta)}^{(x, j, \alpha)} \delta \Phi^{(y, l, \beta)}+\int \mathcal{D}_{((z, \sigma))}^{(x, j, \alpha)} \delta \Omega^{((z, \sigma))}+h^{(x, j, \alpha)}
$$

where

$$
\mathcal{D}_{((z, \sigma))}^{(x, j, \alpha)}=\left(\mathcal{D}_{j}\right)^{\alpha}{ }_{\sigma} \delta^{3}(x-z),
$$

and where the vector $h^{(x, j, \alpha)}$ satisfies the harmonicity conditions, which we again call non-Abelian closed and non-Abelian co-closed, respectively:

$$
\begin{aligned}
& \int\left(\mathcal{D}^{\dagger}\right)_{(x, j, \alpha)}^{(z, \sigma))} h^{(x, j, \alpha)}=0, \\
& \int\left(L^{\dagger}\right)_{(x, j, \alpha)}^{(y, l, \beta)} h^{(x, j, \alpha)}=0 .
\end{aligned}
$$

If there is a second variation $\delta A^{(x, j, \alpha)}$, of $A^{(x, j, \alpha)}$, we decompose it as

$$
\delta A^{\prime(x, j, \alpha)}=\int L_{(y, l, \beta)}^{(x, j, \alpha)} \delta \Phi^{\prime(y, l, \beta)}+\int \mathcal{D}_{((z, \sigma))}^{(x, j, \alpha)} \delta \Omega^{\prime((z, \sigma))}+h^{\prime(x, j, \alpha)},
$$

which yields the decomposition of the inner product:

$$
\begin{aligned}
\int \delta A^{\prime(x, j, \alpha)} \delta A^{(x, j, \alpha)}=\int G_{(u, l, \alpha)(v, m, \beta)} \delta \Phi^{\prime(u, l, \alpha)} \delta \Phi^{(v, m, \beta)} & +\int\left(-\mathcal{D}^{2}\right)_{((z, \gamma))((w, \rho))} \delta \Omega^{\prime((z, \gamma))} \delta \Omega^{((w, \rho))} \\
& +\int h^{\prime(x, j, \alpha)} h^{(x, j, \alpha)}
\end{aligned}
$$

The quantity $\delta \Phi^{(y, \beta)}$ produces a variation of the gauge field which is orthogonal to gauge transformations.

We do not prove here that the dimension of the space of square-integrable harmonic forms is finite, but this statement seems obvious. For the conditions (2.10) and (2.11) or (2.14) and (2.15) hold if and only

$$
\int \diamond_{(y, k, \omega)}^{(x, j, \gamma)} h^{(y, k, \omega)}=0, \diamond=\mathcal{D} \mathcal{D}^{\dagger}+L L^{\dagger}
$$

The operator $\diamond$ is a generalization of the Laplacian on one-forms. This operator should have a pseudo-elliptic self-adjoint extension with a finite number of zero eigenvectors. 
We close this section with the following remark. The second term in the linear transformation $L$ in two (2.3) or three (2.5) space dimensions has the form of a gauge transformation. By a suitable redefinition

$$
\delta \Omega \longrightarrow \delta \Omega^{\prime}=\delta \Omega+\mathrm{i} \frac{1}{\mathcal{D}^{2}} \mathcal{F}_{12} \delta \Phi
$$

in two dimensions and

$$
\delta \Omega \longrightarrow \delta \Omega^{\prime}=\delta \Omega+\mathrm{i} \frac{1}{\mathcal{D}^{2}}(* \mathcal{F}) \cdot \delta \Phi,
$$

in three dimensions, this term can be removed. Then

$$
\begin{gathered}
\delta A^{(x, j, \alpha)}=\int \epsilon^{j k} \mathcal{D}_{(y, \beta)}^{(x, k, \alpha)} \delta \Phi^{(y, \beta)}+\int \mathcal{D}_{((z, \sigma))}^{(x, j, \alpha)} \delta \Omega^{\prime((z, \sigma))}+h^{(x, j, \alpha)}, \\
\delta A^{(x, j, \alpha)}=\int \epsilon^{j k l} \mathcal{D}_{(y, \beta)}^{(x, k, \alpha)} \delta \Phi^{(y, l, \beta)}+\int \mathcal{D}_{((z, \sigma))}^{(x, j, \alpha)} \delta \Omega^{\prime((z, \sigma))}+h^{(x, j, \alpha)},
\end{gathered}
$$

in two and three space dimensions, respectively. These relations (2.19) and (2.20), though much simpler, are not as useful from the point of view of the geometry of orbit space as (2.3) and (2.5), since the splitting into gauge transformations and non-gauge variations is lost. This does not mean they are not interesting, however, and we shall discuss (2.19) again in Section 8.

\section{Hypersurfaces in connection space}

To see the full implication of our decomposition formulas (2.9) and (2.13) for gaugetheory orbit space, we compare them to the formula for the coordinates of a flat space and the coordinates of a hypersurface embedded in this flat space:

$$
d y^{a}=e_{\mu}^{a} d x^{\mu}+n_{H}^{a} d w^{H} .
$$

Here the flat space has Cartesian coordinates $y^{a}, a=1, \ldots, n$, the hypersurface has coordinates $x^{\mu}, \quad \mu=1, \ldots, m<n$ in a particular chart and $w^{H}, \quad H=1, \ldots, n-m$ are coordinates parametrizing the direction normal to the hypersurface. The analogy we make is that orbit space $\mathcal{M}$ is a hypersurface in connection space The hypersurface coordinates are $\{\Phi\}$ and the normal coordinates are $\{\Omega\}$. The tangent-space basis vectors $e_{\mu}^{a}$ and the normal vector basis $n_{H}^{a}$ in $\mathbb{R}^{n}$ correspond in $\mathbb{A}$ to $L$ and $\mathcal{D}$ respectively. Notice that the form of (2.9) and (2.13) implies that the torsion is zero, just as for (3.1). Thus, except at singularities, our coordinates $\{\Phi\}$ are Riemannian.

The coordinates $\{\Phi\}$ we have chosen on gauge orbits are by definition a gauge choice; as is any parametrization of gauge orbits. They differ from traditional gauge choices, e.g. Landau, Feynman, Coulomb, Axial, etc., in that variations in the gauge field are covariantly divergenceless. In Coulomb gauge $\partial_{j} A^{(x, j, \alpha)}$ in two space dimensions, for 
example, we may write $A^{(x, j, \alpha)}=\epsilon^{j k} \partial_{k} \phi_{(x, \alpha)}$. A variation $\delta \phi_{(x, \alpha)}$ typically produces a change in $A^{(x, j, \alpha)}$ which is gauge dependent. This is because $\mathcal{D}_{j} \epsilon^{j k} \partial_{k} \delta \phi_{(x, \alpha)} \neq 0$, in general. In contrast, by virtue of (2.12) and (2.16), any variation in our coordinates

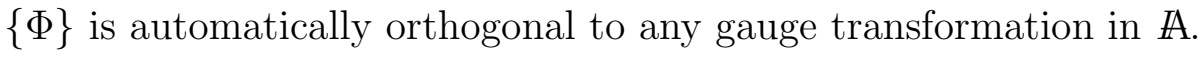

If boundary conditions can be chosen with no harmonic forms, i.e. $h$ satisfying $\diamond h=0$, the analogy between (2.9) and (2.13) and (3.1) is complete. This can be done for most gauge orbits by chosing the boundary conditions appropriately. At particular gauge orbits, however, harmonic forms will appear. Harmonic forms in (2.9) or (2.13) are special gauge transformations. The appearance of a harmonic form means that the dimension of the space of infinitesimal gauge transformations increases by one, and the dimension of infinitesimal non-gauge variations of the connection decreases by one. Thus harmonic forms appear at values of $\{\Phi\}$ at the boundary of our coordinate chart. A subset of this boundary is the Gribov horizon. The values of a connected region of $\{\Phi\}$ excluding the boundary are the coordinates of the fundamental region of orbit space.

If the functions describing the embedding of a hypersurface (3.1) are sufficiently smooth, then we may write the differential-geometric formulas

$$
\partial_{\mu} e_{\nu}^{a}=\Gamma_{\mu \nu}^{\lambda} e_{\lambda}^{a}+\mathrm{K}_{\mu \nu}^{H} n_{H}^{a}, \partial_{\mu} n_{H}^{a}=-g^{\alpha \nu} q_{H J} \mathrm{~K}_{\mu \alpha}^{J} e_{\nu}^{a}+\Xi_{\mu H}^{I} n_{I}^{a},
$$

where $q_{H J}=\sum_{a} n_{H}^{a} n_{J}^{a}$. We recognize $\Gamma_{\mu \nu}^{\lambda}$ as the Riemannian affine connection and $\mathrm{K}_{\mu \nu}^{H}$ as the second fundamental form or extrinsic curvature.

If we ignore the issue of harmonic forms for the time being, it is sensible to ask what relations are analogous to (3.2) for our hypersurface in A. For the case of twodimensional and three-dimensional gauge fields, these are

$$
\begin{aligned}
\frac{\delta L_{(u, j, \kappa)}^{(u, \gamma)}}{\delta \Phi^{(x, \alpha)}} & =\int \Gamma_{(v, \gamma)(x, \alpha)}^{(z, \rho)} L_{(z, \rho)}^{(u, j, \kappa)}+\int \mathrm{K}_{(v, \gamma)(x, \alpha)}^{((t, \sigma))} \mathcal{D}_{((t, \sigma))}^{(u, j, \kappa)}, \\
\frac{\delta \mathcal{D}_{(u, j, \kappa)}^{((s, \tau))}}{\delta \Phi^{(x, \alpha)}} & =-\int G^{(w, \alpha)(z, \gamma)} Q_{((s, \tau))((t, \sigma))} \mathrm{K}_{(r, \mu)(w, \alpha)}^{((t, \sigma))} L_{(z, \gamma)}^{(u, j, \kappa)} \\
& +\int \Xi_{(r, \mu)((s, \tau))}^{((t, \sigma))} \mathcal{D}_{((t, \sigma))}^{(u, j, \kappa)},
\end{aligned}
$$

and

$$
\begin{aligned}
\frac{\delta L^{(u, j, \kappa)}(v, k, \gamma)}{\delta \Phi^{(x, l, \alpha)}} & =\int \Gamma_{(v, k, \gamma)(x, l, \alpha)}^{(z, n, \rho)} L_{(z, n, \rho)}^{(u, j, \kappa)}+\int \mathrm{K}_{(v, k, \gamma)(x, l, \alpha)}^{((t, \sigma))} \mathcal{D}_{((t, \sigma))}^{(u, j, \kappa)} \\
\frac{\delta \mathcal{D}^{(u, j, \kappa)}((s, \tau))}{\delta \Phi^{(x, l, \alpha)}} & =-\int G^{(w, m, \beta)(z, k, \gamma)} Q_{((s, \tau))((t, \sigma))} \mathrm{K}_{(x, l, \alpha)(w, m, \beta)}^{(t, \sigma))} L_{(z, k, \gamma)}^{(u, j, \kappa)} \\
& +\int \Xi_{(x, l, \alpha)((s, \tau))}^{((t, \sigma))} \mathcal{D}_{((t, \sigma))}^{(u, j, \kappa)},
\end{aligned}
$$

respectively, where

$$
Q_{((s, \tau))((t, \sigma))}=\int \mathcal{D}_{((s, \tau))}^{(u, j, \kappa)} \mathcal{D}_{((t, \sigma))}^{(u, j, \kappa)}=\left(-\mathcal{D}^{2}\right)_{((s, \tau))((t, \sigma))}
$$




\section{Gauge-invariant or gauge-covariant?}

We have not yet worked explicitly with the components of $\{\Phi\}$, but only its variation. An explicit expression for these components is given in Section 8, though only for the $(2+1)$-dimensional case. We have claimed that $\{\Phi\}$ constitutes a set of gauge-invariant coordinates (if we chose the domain of $\{\Phi\}$ to be the fundamental region) on orbit space. Though this claim is very easy to justify, it is so crucial to the interpretation of this paper that we felt it necessary to provide the justification.

Note that the metric tensors (2.7) and (2.9) are formally gauge-covariant expressions, and not gauge-invariant. Thus $\delta \Phi$ is gauge-covariant as well. Under a gauge transformation $g(x) \in \mathrm{SU}(N)$ with adjoint representation $D_{\mathrm{ADJ}}(g(x))$,

$$
\delta \Phi^{(x, \alpha)} \rightarrow \delta \Phi^{\prime(x, \alpha)}=D_{\mathrm{ADJ}}(g(x))^{\alpha}{ }_{\beta} \delta \Phi^{(x, \beta)},
$$

in $2+1$ dimensions and

$$
\delta \Phi^{(x, k, \alpha)} \rightarrow \delta \Phi^{\prime(x, k, \alpha)}=D_{\mathrm{ADJ}}(g(x))^{\alpha}{ }_{\beta} \delta \Phi^{(x, k, \beta)},
$$

in $3+1$ dimensions. To properly define $\{\Phi\}$, however, it is necessary to integrate these expressions. To do this, a particular orbit, chosen by taking some particular connection $A^{(x, j, \alpha)}$ is identified with a particular choice of $\{\Phi\}$, say $\{\phi\}$. This choice is a boundary condition on the first-order functional differential equation

$$
\delta A^{(x, j, \alpha)}=\int L_{(y, \beta)}^{(x, j, \alpha)} \delta \Phi^{(y, \beta)},
$$

in two space dimensions or

$$
\delta A^{(x, j, \alpha)}=\int L_{(y, l, \beta)}^{(x, j, \alpha)} \delta \Phi^{(y, l, \beta)},
$$

in three space dimensions. These variations remain on the hypersurface. A gauge transformation changes the boundary condition, and thereby changes the hypersurface. Therefore, we can assign a choice of $\{\Phi\}$ and the gauge field (in the fundamental region) to each orbit (except on a set of measure zero). This is why our coordinates are gaugeinvariant, not gauge-covariant.

It is important that $\mathcal{D}^{2}$ not have zero modes. We can achieve this by putting the system in a finite volume, say a two-dimensional disk or three-dimensional ball. At the boundary, we set all components of $\{\phi\}$ and $\{\delta \Phi\}$ equal to zero. The gauge connection is defined by integrating (4.3) or (4.4). Then the operator manipulations we have performed remain legitimate.

\section{The extrinsic and intrinsic curvatures of the hy- persurface}

We shall next use equations (3.3) and (3.4) to obtain the second fundamental form and the intrinsic curvature of orbit space. The curvature tensor was first discussed 
many years ago [2, 3]. Knowledge of the intrinsic curvature can give important global information relevant to the spectrum of the Laplacian [15].

For the case of a hypersurface in finite-dimensional space, the equations (3.2) imply that

$$
\mathrm{K}_{H, \mu \nu}=q_{H J} \mathrm{~K}_{\mu \nu}^{J}=-\sum_{a} e_{\mu}^{a} \partial_{\nu} n_{H}^{a}
$$

The curvature tensor is given in terms of the second fundamental form by Gauss' formula:

$$
R_{\alpha \beta \mu \nu}=q^{H J}\left[\mathrm{~K}_{H, \alpha \mu} \mathrm{K}_{J, \beta \nu}-\mathrm{K}_{H, \alpha \beta} \mathrm{K}_{J, \mu \nu}\right]
$$

where $q^{H J}$ is the inverse of $q_{H J}$.

We first consider the second fundamental form for $(2+1)$-dimensional gauge theories. The generalization of (5.1) to the hypersurface in connection space is

$$
\begin{aligned}
\mathrm{K}_{((z, \lambda)),(x, \alpha)(y, \beta)} & =-\int L_{(x, \alpha)}^{(u, j, \gamma)} \frac{\delta \mathcal{D}_{(u, j, \gamma)}^{((z, \lambda))}}{\delta \Phi^{(y, \beta)}}=-\int L_{(x, \alpha)}^{(u, j, \gamma)} L_{(y, \beta)}^{(v, k, \sigma)} \frac{\delta \mathcal{D}_{(u, j, \gamma)}^{(u, \lambda))}}{\delta A^{(v, k, \sigma)}} \\
& =-f_{\lambda \gamma \sigma} L_{(x, \alpha)}^{(z, j, \gamma)} L_{(y, \beta)}^{(z, j, \sigma)},
\end{aligned}
$$

where in the last expression the sum is implicit on $j, \gamma$ and $\sigma$. For $3+1$ dimensions

$$
\mathrm{K}_{((z, \lambda)),(x, l, \alpha)(y, m, \beta)}=-f_{\lambda \gamma \sigma} L_{(x, l, \alpha)}^{(z, j, \gamma)} \underset{(y, m, \beta)}{(z, j, \sigma)} .
$$

Notice that there is no integration in the final form of either of these expressions.

The curvature (5.2) generalized to $(2+1)$-dimensional gauge theories is

$$
\begin{aligned}
R_{(x, \alpha)(y, \beta)(u, \mu)(v, \nu)} & =\int\left[\left(\frac{1}{-\mathcal{D}^{2}}\right)^{\lambda \rho} \delta^{2}(z-w)\right]\left[\mathrm{K}_{((z, \lambda)),(x, \alpha)(u, \mu)} \mathrm{K}_{((w, \rho)),(y, \beta)(v, \nu)}\right. \\
& \left.-\mathrm{K}_{((z, \lambda)),(x, \alpha)(y, \beta)} \mathrm{K}_{((w, \rho)),(u, \mu)(v, \nu)}\right] .
\end{aligned}
$$

An expression similar to (5.5) can be found in reference [3], but the second fundamental form was not explicitly determined there. Inserting (5.3) into (5.5) yields

$$
\begin{aligned}
R_{(x, \alpha)(y, \beta)(u, \mu)(v, \nu)} & \int\left[\left(\frac{1}{-\mathcal{D}^{2}}\right)^{\lambda \rho} \delta^{2}(z-w)\right] f_{\lambda \gamma \sigma} f_{\rho \kappa \tau} \\
\times & {\left[L_{(x, \alpha)}^{(z, j, \gamma)} L_{(u, \mu)}^{(z, j, \sigma)} L_{(y, \beta)}^{(w, k, \kappa)} L_{(v, \nu)}^{(w, k, \tau)}\right.} \\
& -L_{(x, \alpha)}^{(z, j, \gamma)} \underset{(y, \beta)}{\left.\stackrel{(z, j, \sigma)}{(u, k, \mu)} L_{(v, \nu)}^{(w, k, \kappa)} L_{(u, k, \tau)}^{(w)}\right] .}
\end{aligned}
$$

The corresponding expression for $(3+1)$-dimensional gauge theories is

$$
R_{(x, l, \alpha)(y, m, \beta)(u, p, \mu)(v, r, \nu)}=\int\left[\left(\frac{1}{-\mathcal{D}^{2}}\right)^{\lambda \rho} \delta^{3}(z-w)\right] f_{\lambda \gamma \sigma} f_{\rho \kappa \tau}
$$




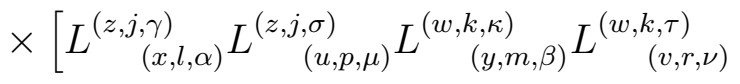

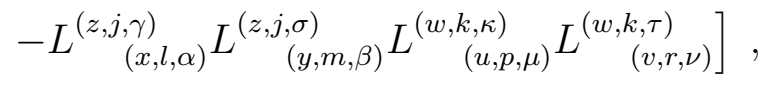

where $l, m, p, r=1,2$. These expressions for the curvature are evidently well-defined with no regularization. This is no longer the case for the contractions of the curvature tensor, namely the Ricci and scalar curvatures.

\section{Sectional, Ricci and scalar curvatures for Yang- Mills theory in arbitrary dimensions}

We will next discuss the curvature tensor of orbit space and its contractions for an arbitrary number of space dimensions $\mathrm{D}$ (so space-time has $\mathrm{D}+1$ dimensions). We anticipate that our expressions for the Ricci and scalar curvatures may be used to calculate these quantities in dimensional regularization. We hope to perform these calculations near particular gauge-field backgrounds in a future publication. We should mention that Singer discussed a calculation of the Ricci curvature using zeta-function regularization [2, though some details were evidently never published.

The non-Abelian exactness condition in D space dimensions, generalizing (2.2) and (2.4), is

$$
\delta A^{(x, j, \alpha)}=L_{\left(y, l_{1} l_{2} \cdots l_{\mathrm{D}-2}, \beta\right)}^{(x, j, \alpha)} \delta \Phi^{\left(y, l_{1} l_{2} \cdots l_{\mathrm{D}-2}, \beta\right)},
$$

where the mapping from the hypersurface to connection space in any space dimension $\mathrm{D}$, generalizing (2.3) and (2.5) is

$$
\begin{aligned}
& L_{\left(y, l_{1} l_{2} \cdots l_{\mathrm{D}-2}, \beta\right)}^{(x, j, \alpha)}=\frac{\delta A^{(x, j, \alpha)}}{\delta \Phi^{\left(y, l_{1} l_{2} \cdots l_{\mathrm{D}-2}, \beta\right)}} \\
& \quad=\left[\epsilon^{j k l_{1} l_{2} \cdots l_{\mathrm{D}-2}} \mathcal{D}_{k}+\mathrm{i} \mathcal{D}_{j} \frac{1}{\mathcal{D}^{2}}(* \mathcal{F})^{\left(x, l_{1} l_{2} \cdots l_{\mathrm{D}-2}\right)}\right]_{\beta}^{\alpha} \delta^{\mathrm{D}}(x-y),
\end{aligned}
$$

The variation $\delta \Phi^{\left(y, l_{1} l_{2} \cdots l_{\mathrm{D}-2}, \beta\right)}$ is fully antisymmetric in the space indices $l_{1}, l_{2}, \ldots, l_{\mathrm{D}-2}$. As we would expect, the field dual to a vector-gauge field is an antisymmetric-tensor gauge field of rank D-2 (in d space-time dimensions, this is d-3). The infinitesimal dual gauge transformations are the zero modes of the mapping $L$.

The metric tensor for $\mathrm{D}>2$ dimensions is not

$$
G_{(u,\{p\}, \mu)(v,\{r\}, \nu)}=\int L_{(u,\{p\}, \mu)}^{(x, j, \alpha)} \underset{(v,\{r\}, \nu)}{(x, j, \alpha)},
$$

but rather

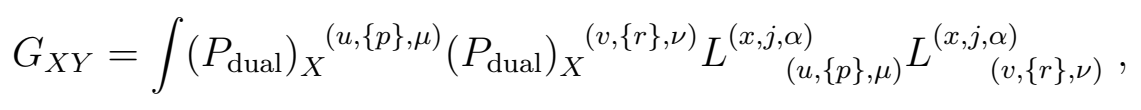


where $\left(P_{\text {dual }}\right)_{X}{ }^{(x,\{l\}, \alpha)}$ is the projection operator which projects out the zero modes of $L$. Here the indices $X, Y$ are those of the subspace with these zero modes removed. This solution of the dual-gauge-invariance problem is more abstract than used earlier in this paper, but is also more convenient for the goal of determining the Ricci and scalar curvatures for arbitrary space dimensions.

Before projecting out the zero modes of $L$, the curvature tensor, determined by the methods of the last section is

$$
\begin{aligned}
& R_{(x,\{l\}, \alpha)(y,\{m\}, \beta)(u,\{p\}, \mu)(v,\{r\}, \nu)}=\int\left[\left(\frac{1}{-\mathcal{D}^{2}}\right)^{\lambda \rho} \delta^{\mathrm{D}}(z-w)\right] f_{\lambda \gamma \sigma} f_{\rho \kappa \tau}
\end{aligned}
$$

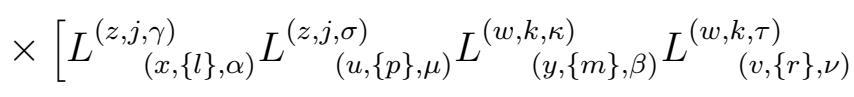

$$
\begin{aligned}
& \left.-L_{(x,\{l\}, \alpha)}^{(z, j, \gamma)} \underset{(y,\{m\}, \beta)}{\stackrel{(z, j, \sigma)}{(w, k, \kappa)}} \underset{(u,\{p\}, \mu)}{\stackrel{(w, k, \tau)}{(v,\{r\}, \nu)}]}\right],
\end{aligned}
$$

where $\{l\}$ is an abbreviation for $l_{1}, l_{2}, \ldots, l_{\mathrm{D}-2}$, etc. Again, some of the components of (6.5) should be removed; this is because of the dual gauge invariance. We do this using projection operators, instead of restricting indices. The actual curvature $\mathcal{R}_{X Y U V}$ for $\mathrm{D}>2$ is given by

$$
\begin{aligned}
\mathcal{R}_{X Y U V} & =\int\left(P_{\text {dual }}\right)_{X}{ }^{(x,\{l\}, \alpha)}\left(P_{\text {dual }}\right)_{Y}{ }^{(y,\{m\}, \beta)}\left(P_{\text {dual }}\right)_{U}{ }^{(u,\{p\}, \mu)}\left(P_{\text {dual }}\right)_{V}{ }^{(v,\{r\}, \nu)} \\
& \times R_{(x,\{l\}, \alpha)(y,\{m\}, \beta)(u,\{p\}, \mu)(v,\{r\}, \nu)}
\end{aligned}
$$

The sectional curvature between (real) vectors $\xi^{X}, \zeta^{X}$ is

$$
\frac{\int \mathcal{R}_{X Y U V} \xi^{X} \xi^{Y} \zeta^{U} \zeta^{V}}{\langle\xi \mid \zeta\rangle^{2}}
$$

where

$$
\langle\xi \mid \zeta\rangle=\int G_{(x,\{l\}, \alpha)(y,\{m\}, \beta)}\left(P_{\text {dual }}\right)_{X}{ }^{(x,\{l\}, \alpha)}\left(P_{\text {dual }}\right)_{Y}{ }^{(x,\{m\}, \beta)} \xi^{X} \zeta^{Y}
$$

Due to the antisymmetry of the group structure coefficients $\{f\}$, the second term of the curvature tensor does not contribute to the numerator of (6.7); hence the sectional curvature is non-negative.

We introduce a second projection operator

$$
P^{(x, j, \alpha)(y, k, \beta)}=\left[\delta_{j k} \delta^{\alpha \beta}-\mathcal{D}_{j} \frac{1}{\mathcal{D}^{2}} \mathcal{D}_{k}\right] \delta^{\mathrm{D}}(x-y)
$$

which has the same form as the metric discussed in the introduction (1.2). This projects out ordinary Yang-Mills gauge transformations. 
To obtain the Ricci curvature, we must contract (i.e. multiply and carry out the sum and integration over repeated indices) the curvature tensor (6.6) with the inverse metric. This is

$$
G^{U V}=\int\left(P_{\text {dual }}\right)_{U}{ }^{(u,\{p\}, \mu)}\left(P_{\text {dual }}\right)_{V}{ }^{(v,\{r\}, \nu)}\left(L^{-1}\right)_{(x, j, \alpha)}^{(u,\{p\}, \mu)}\left(L^{-1}\right)_{(y, k, \beta)}^{(v,\{r\}, \nu)} P^{(x, j, \alpha)(y, k, \beta)}
$$

We have inserted $P$ and $P_{\text {dual }}$ into (6.10) to keep this expression well-defined.

We are finally ready to write down the Ricci tensor. We contract the curvature tensor (6.6) with (6.10) to obtain

$$
\operatorname{Ric}_{X Y}=\int\left(P_{\text {dual }}\right)_{X}{ }^{(x,\{l\}, \alpha)}\left(P_{\text {dual }}\right)_{Y}{ }^{(y,\{m\}, \beta)} \operatorname{Ric}_{(x,\{l\}, \alpha)(y,\{m\}, \beta)},
$$

where

$$
\begin{aligned}
\operatorname{Ric}_{(x,\{l\}, \alpha)(y,\{m\}, \beta)} & =\int\left[\left(\frac{1}{-\mathcal{D}^{2}}\right)^{\lambda \rho} \delta^{\mathrm{D}}(z-w)\right] f_{\lambda \gamma \sigma} f_{\rho \kappa \tau} \\
& \times L_{(x,\{l\}, \alpha)}^{(z, j, \gamma)} \underset{(y,\{m\}, \beta)}{(w, k, \kappa)} P_{(z, j, \sigma)(w, k, \tau)} .
\end{aligned}
$$

Notice that the Ricci curvature is formally non-negative. Once this expression is regularized, it will be important to see if a positive lower bound exists on its spectrum.

The scalar curvature is obtained by a further contraction of the Ricci curvature with the inverse metric. It is comparatively simple, containing no factors of $P_{\text {dual }}$ :

$$
\mathrm{R}=\int\left[\left(\frac{1}{-\mathcal{D}^{2}}\right)^{\lambda \rho} \delta^{\mathrm{D}}(z-w)\right] f_{\lambda \gamma \sigma} f_{\rho \kappa \tau} P_{(z, j, \gamma)(w, k, \kappa)} P_{(z, j, \sigma)(w, k, \tau)}
$$

As mentioned at the beginning at the section, we hope to study these quantities further in a later publication. Our intention is to expand the Green's functions in (6.12) and (6.13) around particular backgrounds with space dimension D close to an integer value.

\section{The Yang-Mills Hamiltonian}

In this section, we will examine the kinetic term of the Hamiltonian of non-Abelian gauge theories in terms of the gauge-invariant coordinates $\{\Phi\}$ and consider the functional form of the measure.

The Yang-Mills Hamiltonian operator is

$$
H=T+U, \quad T=-\frac{e_{0}^{2}}{2} \int \frac{\delta^{2}}{\delta A^{(x, j, \alpha)} \delta A^{(x, j, \alpha)}}, \quad U=\frac{1}{2 e_{0}^{2}} \int\left(F_{j k}\right)^{2},
$$

and wave functions depend only on $\Phi$ by Gauss' law. The coupling constant $e_{0}$ has engineering dimension $(4-d) / 2$, where $d$ is the dimension of space-time. 
The kinetic term in $2+1$ dimensions may be written as the Laplacian (we use $e$ rather than $e_{0}$, since the theory is finite)

$$
T=-\frac{e^{2}}{2} \int \frac{1}{\sqrt{\operatorname{det} G}} \frac{\delta}{\delta \Phi^{(y, \beta)}} \sqrt{\operatorname{det} G} G^{(y, \beta)(z, \gamma)} \frac{\delta}{\delta \Phi^{(z, \gamma)}},
$$

where the $G_{(x, \alpha)(y, \beta)}$ and $G^{(x, \alpha)(y, \beta)}$ are the component of the metric tensor (2.7) and its inverse, respectively, on the hypersurface. In $3+1$ dimensions

$$
T=-\frac{e_{0}^{2}}{2} \int \frac{1}{\sqrt{\operatorname{det} G}} \frac{\delta}{\delta \Phi^{(y, l, \beta)}} \sqrt{\operatorname{det} G} G^{(y, l, \beta)(z, m, \gamma)} \frac{\delta}{\delta \Phi^{(z, m, \gamma)}},
$$

with $l, m=1,2$. Alternative expressions can be given in by chosing different dual gauge fixings.

The inner product on Schrödinger wave functionals is the functional integral

$$
\left\langle\Psi_{1} \mid \Psi_{2}\right\rangle=\int[d \Phi] \sqrt{\operatorname{det} G} \bar{\Psi}_{1}[\Phi] \Psi_{2}[\Phi] .
$$

It is the measure factor in this inner product which is responsible for the appearance of the mass gap [9, 10, as we discuss in Section 10.

Strictly speaking the Hamiltonian may have another term equal to $\frac{\mathrm{R}}{6}$ where $\mathrm{R}$ is the scalar curvature (6.13). It was pointed out by Gawedzki [16] that such a term can be present. If one begins with (7.1) and (7.2) or (7.2) and uses the Trotter product formula [17] to determine the action principle one finds a Euclidean action

$$
S=\int \frac{1}{2 e_{0}^{2}} \dot{\Phi}^{T} G \dot{\Phi}+\int d \tau \frac{\mathrm{R}}{6}+\int \frac{1}{4 e_{0}^{2}} \operatorname{Tr} F_{i j} F_{i j},
$$

where $T$ denotes the transpose, $\tau$ denotes Euclidean time and the dot denotes timedifferentiation. Removal of the scalar-curvature term from the action will force this term into the Hamiltonian. It is not yet clear how important this term is; it may be irrelevant (in the renormalization group sense) in which case it may be dropped. If the term is not irrelevant, however, it may be essential for a Lorentz-invariant spectrum. We leave this question open in the remaining of this paper, though we believe that it can be answered by the calculation we have proposed at the end of the last section.

\section{Solving for $\{\Phi\}$ in $2+1$ dimensions}

A drawback of our formalism is that we can only write the variation of the gauge field in terms of the variation of $\{\Phi\}$. We would like an expression for $A^{(x, j, \alpha)}$ in terms of $\Phi^{(y, \lambda)}$. Though we have not yet solved this problem, we have done the reverse by finding an expression for $\Phi^{(y, \lambda)}$ in terms of $A^{(x, j, \alpha)}$ in two space dimensions.

We define holomorphic coordinates $z=x^{1}+\mathrm{i} x^{2}, \bar{z}=x^{1}-\mathrm{i} x^{2}$ and their derivatives $\partial=\frac{1}{2}\left(\partial_{1}-\mathrm{i} \partial_{2}\right), \bar{\partial}=\frac{1}{2}\left(\partial_{1}+\mathrm{i} \partial_{2}\right)$ (we are no longer using the letter $z$ to represent 
a Cartesian coordinate of space, as we did in earlier sections). The gauge field in the fundamental representation has the components $A^{(z, \bar{z}, \alpha)}=\frac{1}{2}\left(A^{(x, 1, \alpha)}-\mathrm{i} A^{(x, 2, \alpha)}\right)$, $\bar{A}^{(z, \bar{z}, \alpha)}=\frac{1}{2}\left(A^{(x, 1, \alpha)}+\mathrm{i} A^{(x, 2, \alpha)}\right)$, in these coordinates. We also define $\mathcal{D}=\frac{1}{2}\left(\mathcal{D}_{1}-\mathrm{i} \mathcal{D}_{2}\right)$, $\overline{\mathcal{D}}=\frac{1}{2}\left(\mathcal{D}_{1}+\mathrm{i} \mathcal{D}_{2}\right)$.

In holomorphic coordinates, equation (2.9) becomes

$$
\begin{gathered}
\delta A^{(z, \bar{z}, \alpha)}=\mathrm{i}\left[\mathcal{D}\left(1+\frac{1}{\mathcal{D} \overline{\mathcal{D}}+\overline{\mathcal{D}} \mathcal{D}}[\mathcal{D}, \overline{\mathcal{D}}]\right)\right]_{\beta}^{\alpha} \delta \Phi^{(z, \bar{z}, \beta)}+\mathcal{D}^{\alpha}{ }_{\beta} \delta \Omega^{(z, \bar{z}, \beta)}+h^{(z, \bar{z}, \alpha)} \\
\delta \bar{A}^{(z, \bar{z}, \alpha)}=-\mathrm{i}\left[\overline{\mathcal{D}}\left(1-\frac{1}{\mathcal{D} \overline{\mathcal{D}}+\overline{\mathcal{D}} \mathcal{D}}[\mathcal{D}, \overline{\mathcal{D}}]\right)\right]_{\beta}^{\alpha} \delta \Phi^{(z, \bar{z}, \beta)}+\overline{\mathcal{D}}^{\alpha}{ }_{\beta} \delta \Omega^{(z, \bar{z}, \beta)} \\
+\bar{h}^{(z, \bar{z}, \alpha)} .
\end{gathered}
$$

If there are no harmonic forms, the equations (8.1) imply the remarkable relation

$$
\delta \Phi=-\frac{\mathrm{i}}{2} \mathcal{D}^{-1} \delta A+\frac{\mathrm{i}}{2} \overline{\mathcal{D}}^{-1} \delta \bar{A}
$$

We would like to integrate (8.2). Notice that $\delta \Phi$ can be regarded as a one-form on infinite-dimensional connection space A. Furthermore, this one-form is closed:

$$
\frac{\delta}{\delta A^{(z, \bar{z}, \alpha)}} \frac{\mathrm{i}}{2} \overline{\mathcal{D}}^{-1}-\frac{\delta}{\delta \bar{A}^{(z, \bar{z}, \beta)}}(-) \frac{\mathrm{i}}{2} \mathcal{D}^{-1}=0+0=0,
$$

except at values of $A, \bar{A}$ where $\mathcal{D}$ or $\overline{\mathcal{D}}$ has a zero mode. Therefore, if some particular choice of $\{\Phi\}$, namely $\{\phi\}$ is identified with the gauge field $\{B\},\{\bar{B}\}$, we have

$$
\Phi=\phi+\int_{\phi}^{\Phi} d \Phi^{\prime}=\phi+\int_{B, \bar{B}}^{A, \bar{A}}\left[-\frac{\mathrm{i}}{2} \frac{1}{\mathcal{D}^{\prime}} d A^{\prime}+\frac{\mathrm{i}}{2} \frac{1}{\overline{\mathcal{D}}^{\prime}} d \bar{A}^{\prime}\right] .
$$

By virtue of (8.3), this integral is independent of the path of integration chosen in $A$, provided the path does not encounter any values of $A^{\prime}, \bar{A}^{\prime}$ where either $\mathcal{D}^{\prime}$ or $\overline{\mathcal{D}}^{\prime}$ has a zero eigenvalue. Furthermore, if the path does not encounter such singularities, $\diamond$ will not have a zero eigenvalue and there are no harmonic forms to worry about. Therefore, the question of whether $\mathcal{D} b=0$ or $\overline{\mathcal{D}} b=0$ has a solution is important. In a finite volume, these solutions will not exist for most gauge fields 18 .

An explicit realization of $(8.4)$ is obtained by taking

$$
\begin{aligned}
A^{\prime} & =A(x, T)=T[A(x)-B(x)]+B(x), \\
\bar{A}^{\prime} & =\bar{A}(x, T)=T[\bar{A}(x)-\bar{B}(x)]+\bar{B}(x),
\end{aligned}
$$

for $0 \leq T \leq 1$. Thus (8.4) becomes, at least formally

$$
\begin{aligned}
& \Phi=\phi-\frac{\mathrm{i}}{2} \int_{0}^{1} d T\left[\frac{1}{\partial-\mathrm{i} T(\mathcal{A}-\mathcal{B})-\mathrm{i} \mathcal{B}}(A-B)\right. \\
& \left.-\frac{1}{\bar{\partial}-\mathrm{i} T(\overline{\mathcal{A}}-\overline{\mathcal{B}})-\mathrm{i} \overline{\mathcal{B}}}(\bar{A}-\bar{B})\right] \text {, }
\end{aligned}
$$


which should be well-defined, provided no zero modes exist for $\partial-\mathrm{i} T(\mathcal{A}-\mathcal{B})-\mathrm{i} \mathcal{B}$ or $\bar{\partial}-\mathrm{i} T(\overline{\mathcal{A}}-\overline{\mathcal{B}})-\mathrm{i} \overline{\mathcal{B}}$ for any $T$ in the closed interval $[0,1]$.

We can make the conclusions above more transparent by making the following redefinition of $\delta \Omega^{(z, \bar{z}, \beta)}$ in the non-Abelian decomposition formula (8.1)

$$
\delta \Omega^{(z, \bar{z}, \alpha)} \longrightarrow \delta \Omega^{\prime(z, \bar{z}, \beta)}=\delta \Omega^{(z, \bar{z}, \alpha)}+\mathrm{i}\left(\frac{1}{\mathcal{D} \overline{\mathcal{D}}+\overline{\mathcal{D}} \mathcal{D}}[\mathcal{D}, \overline{\mathcal{D}}]\right)_{\beta}^{\alpha} \delta \Phi^{(z, \bar{z}, \beta)}
$$

With this redefinition (8.1) becomes

$$
\begin{gathered}
\delta A^{(z, \bar{z}, \alpha)}=\mathrm{i} \mathcal{D}^{\alpha}{ }_{\beta} \delta \Phi^{(z, \bar{z}, \beta)}+\mathcal{D}^{\alpha}{ }_{\beta} \delta \Omega^{\prime(z, \bar{z}, \beta)}+h^{(z, \bar{z}, \alpha)}, \\
\delta \bar{A}^{(z, \bar{z}, \alpha)}=-\mathrm{i} \overline{\mathcal{D}}^{\alpha}{ }_{\beta} \delta \Phi^{(z, \bar{z}, \beta)}+\overline{\mathcal{D}}^{\alpha}{ }_{\beta} \delta \Omega^{\prime(z, \bar{z}, \beta)}+\bar{h}^{(z, \bar{z}, \alpha)} .
\end{gathered}
$$

Notice that (8.6) is (2.17) and (8.7) is (2.19) in holomorphic coordinates. It is easy to check that replacing (8.1) by (8.7) does not affect (8.2) through (8.5). This is because

these are gauge-invariant expressions, hence unaffected by the gauge transformation (8.6).

\section{More about the $(2+1)$-dimensional Hamiltonian}

We now reexamine the form of the kinetic term of the Hamiltonian of a non-Abelian gauge theory in $2+1$ dimensions, briefly discussed in Section 7 , in the light of the result (8.2). Our expressions are very similar those in references [9, 10].

The Yang-Mills Hamiltonian operator in two space dimensions is

$$
H=T+U, \quad T=-\frac{e^{2}}{2} \int \frac{\delta^{2}}{\delta A^{(x, \alpha)} \delta A^{(x, \alpha)}}, \quad U=\frac{1}{2 e^{2}} \int\left(F_{12}\right)^{2},
$$

and wave functionals depend only on $\Phi$. The coupling constant $e$ has engineering dimension one-half.

The kinetic term may be written with the aid of (8.2) as the Laplacian

$$
T=-\frac{e^{2}}{2} \int \frac{1}{\sqrt{G}} \frac{\delta}{\delta \Phi^{(y, \beta)}} \sqrt{G} G^{(y, \beta)(z, \gamma)} \frac{\delta}{\delta \Phi^{(z, \gamma)}},
$$

where the inverse metric tensor on the hypersurface is

$$
G^{(y, \beta)(z, \gamma)}=\left[\frac{1}{\mathcal{D}} \frac{1}{\overline{\mathcal{D}}}\right]^{\beta \gamma} \delta^{2}(y-z) .
$$

This tensor is therefore the inverse of (2.7) after a change of coordinates. Therefore, the metric tensor now reads

$$
G_{(y, \beta)(z, \gamma)}=[\mathcal{D} \overline{\mathcal{D}}]_{\beta \gamma} \delta^{2}(y-z) .
$$

The inner product on Schrödinger wave functionals is the functional integral

$$
\left\langle\Psi_{1} \mid \Psi_{2}\right\rangle=\int[d \Phi] \sqrt{\operatorname{det}[\mathcal{D} \overline{\mathcal{D}}]} \bar{\Psi}_{1}[\Phi] \Psi_{2}[\Phi] .
$$

The kinetic term in equations (8.2a-e) of the first paper of reference [10] closely resembles (9.2). It will be important to fully understand the connection. 


\section{The dual Hamiltonian and the mass gap}

The origin of the Yang-Mills mass gap in the picture put forth in references [9, 10, 13] is in the functional measure in the inner product. We shall briefly discuss this in the context of a dual Hamiltonian formulation. We show only in principle how the gap can be obtained; the complete calculation is under study.

We first discuss quantum mechanics in curved space. Consider an n-dimensional manifold without boundary with coordinates $x^{\mu}, \mu=1, \ldots, n$ and metric tensor $g_{\mu \nu}$. The inner product is

$$
\left\langle\Psi_{1} \mid \Psi_{2}\right\rangle=\int d^{n} x \sqrt{\operatorname{det} g} \bar{\Psi}_{1}(x) \Psi_{2}(x),
$$

and the matrix element of minus the Laplace operator is

$$
\begin{aligned}
\left\langle\Psi_{1}|-\Delta| \Psi_{2}\right\rangle= & -\int d^{n} x \sqrt{\operatorname{det} g} \bar{\Psi}_{1}(x) \frac{1}{\sqrt{\operatorname{det} g}} \partial_{\mu} \sqrt{\operatorname{det} g} g^{\mu \nu} \partial_{\nu} \Psi_{2}(x) \\
& =\int d^{n} x \sqrt{\operatorname{det} g} g^{\mu \nu} \partial_{\mu} \bar{\Psi}_{1}(x) \partial_{\nu} \Psi_{2}(x),
\end{aligned}
$$

Next we introduce $\psi_{1,2}(x)=(\operatorname{det} g)^{\frac{1}{4}} \Psi_{1,2}(x)$, so that (10.1) has the standard flatspace form

$$
\left\langle\Psi_{1} \mid \Psi_{2}\right\rangle=\int d^{n} x \bar{\psi}_{1}(x) \psi_{2}(x)
$$

and (10.2) becomes

$$
\begin{aligned}
\left\langle\Psi_{1}|-\Delta| \Psi_{2}\right\rangle & =\int d^{n} x g^{\mu \nu}\left[\left(\partial_{\mu}-\frac{1}{4} \partial_{\mu} \operatorname{Tr} \log g\right) \bar{\psi}_{1}(x)\right]\left[\left(\partial_{\nu}-\frac{1}{4} \partial_{\nu} \operatorname{Tr} \log g\right) \psi_{2}(x)\right] \\
& =\int d^{n} x\left\{g^{\mu \nu}\left[\partial_{\mu} \bar{\psi}_{1}(x)\right]\left[\partial_{\nu} \psi_{2}(x)\right]+W(x) \bar{\psi}_{1}(x) \psi_{2}(x)\right\}
\end{aligned}
$$

where in the last step we integrated by parts and

$$
\begin{aligned}
W(x) & =\frac{1}{4} \partial_{\mu}\left(g^{\mu \nu} \partial_{\nu} \operatorname{Tr} \log g\right)+\frac{1}{16} g^{\mu \nu}\left(\partial_{\mu} \operatorname{Tr} \log g\right)\left(\partial_{\nu} \operatorname{Tr} \log g\right) \\
& =(\operatorname{det} g)^{-\frac{1}{4}} \partial_{\mu}\left[g^{\mu \nu} \partial_{\nu}(\operatorname{det} g)^{\frac{1}{4}}\right] .
\end{aligned}
$$

Consider now a quantum-mechanical Hamiltonian $H=-\Delta+U(x)$. In the neighborhood of a point of the manifold where $g_{\mu \nu}=\delta_{\mu \nu}$, we can expand $g^{\mu \nu}=\delta^{\mu \nu}+\cdots$, the function $W(x)$ can be interpreted as a correction to the potential energy $U(x)$. This is analogous to what is done in references [9, 10, 13] in their coordinates and what we shall do in our own coordinates. The only difference is that the manifold has an infinite number of dimensions. We define the effective Hamiltonian in finite dimensions as the operator with matrix elements

$$
\left\langle\psi_{1}\left|H_{\mathrm{eff}}\right| \psi_{2}\right\rangle=\int d^{n} x\left\{\frac{1}{2} g^{\mu \nu}\left[\partial_{\mu} \bar{\psi}_{1}(x)\right]\left[\partial_{\nu} \psi_{2}(x)\right]+\left[\frac{1}{2} W(x)+U(x)\right] \bar{\psi}_{1}(x) \psi_{2}(x)\right\}
$$


where $\psi_{1}$ and $\psi_{2}$ are the square-integrable "wave functions" with inner product (10.3).

Next we write down the analogue of (10.6) for Yang-Mills theory in 2+1 dimensions. We write the metric (2.7) as

$$
G_{(x, \alpha)(y, \beta)}=-\partial^{2} \delta_{\alpha \beta} \delta^{2}(x-y)+\left[\left(-\mathcal{D}^{2}+\partial^{2} \mathbb{I}\right)+\mathcal{F}_{12} \frac{1}{-\mathcal{D}^{2}} \mathcal{F}_{12}\right]_{\alpha \beta} \delta^{2}(x-y),
$$

and its inverse as an expansion

$$
\begin{aligned}
& G^{(x, \alpha)(y, \beta)}=\frac{1}{-\partial^{2}} \delta^{\alpha \beta} \delta^{2}(x-y) \\
& \quad-\left(\frac{1}{-\partial^{2}}\right)\left[\left(-\mathcal{D}^{2}+\partial^{2} I I\right)+\mathcal{F}_{12} \frac{1}{-\mathcal{D}^{2}} \mathcal{F}_{12}\right]_{\alpha \beta}\left(\frac{1}{-\partial^{2}}\right) \delta^{2}(x-y)+\cdots,
\end{aligned}
$$

where $(\mathbb{I I})_{\alpha \beta}=\delta_{\alpha \beta}$. We introduce a new physical field $\mathcal{Y}^{\alpha}=\left(-\partial^{2}\right)^{1 / 2} \Phi^{\alpha}$. We assume that $\Phi^{\alpha}=\delta \Phi^{\alpha}$ is close to zero and identify zero gauge field with zero $\Phi$. In this approximation the magnetic field is

$$
\begin{aligned}
\mathcal{F}_{12} & =\delta \mathcal{F}_{12}=\mathcal{D}_{1} \delta A_{2}-\mathcal{D}_{2} \delta A_{1}=\left(-\mathcal{D}^{2}-\mathcal{F}_{12} \frac{1}{-\mathcal{D}^{2}} \mathcal{F}_{12}\right) \delta \Phi \\
& =\left(-\mathcal{D}^{2}-\mathcal{F}_{12} \frac{1}{-\mathcal{D}^{2}} \mathcal{F}_{12}\right)\left(-\partial^{2}\right)^{-1 / 2} \mathcal{Y}=\left(-\partial^{2}\right)^{1 / 2} \mathcal{Y}+\cdots
\end{aligned}
$$

The effective Hamiltonian takes the form

$$
H_{\mathrm{eff}}=\int\left\{-\frac{e^{2}}{2} \frac{\delta^{2}}{\left(\delta \mathcal{Y}^{\alpha}\right)^{2}}+\frac{e^{2}}{2} W[\mathcal{Y}]+\frac{1}{2 e^{2}}\left(\partial_{j} \mathcal{Y}^{\alpha}\right)^{2}+\cdots\right\}
$$

where the higher-order terms can be found from (10.7) and (10.8) and

$$
W[\mathcal{Y}]=(\operatorname{det} G)^{-\frac{1}{4}} \frac{\delta}{\delta \Phi^{(x, \alpha)}}\left[G^{(x, \alpha)(y, \beta)} \frac{\delta}{\delta \Phi^{(y, \beta)}}(\operatorname{det} G)^{\frac{1}{4}}\right] .
$$

The leading terms of the effective Hamiltonian (10.9) resemble an ordinary scalar field theory. The gap is a term of the form $\left(\mathcal{Y}^{\alpha}\right)^{2}$ in $W[\mathcal{Y}]$. Its existence depends on the form of the regularized determinant of the metric in (10.10).

An expression for the effective Hamiltonian can also be obtained in $3+1$ dimensions. The leading terms are those of an Abelian gauge theory, instead of a scalar field theory. The appearance of the gap would be a signal of the breaking of dual gauge symmetry.

We write the three-dimensional metric tensor as

$$
\begin{aligned}
& G_{(x, l, \alpha)(y, m, \beta)}=\left(-\partial^{2} \delta_{l m}-\partial_{l} \partial_{m}\right) \delta_{\alpha \beta} \delta^{3}(x-y) \\
& \quad+\left[\left(-\mathcal{D}^{2}+\partial^{2} \amalg \delta^{l m}\right)+\left(\mathcal{D}_{l} \mathcal{D}_{m}-\partial_{l} \partial_{m} \amalg\right)-\mathrm{i} \mathcal{F}_{l m}+(* \mathcal{F})_{l} \frac{1}{-\mathcal{D}^{2}}(* \mathcal{F})_{m}\right]_{\alpha \beta} \delta^{3}(x-y) .
\end{aligned}
$$


There are several ways to dual-gauge fix, thereby removing the zero mode of the first term of the right-hand side of (10.11). For example, taking a dual-Coulomb gauge $\partial_{l} \Phi^{(x, l, \alpha)}=0$ will lead to the expansion of the inverse metric tensor beginning as

$$
G^{(x, l, \alpha)(y, m, \beta)}=\left(\frac{1}{-\partial^{2}}\right) \delta^{\alpha \beta} \delta^{3}(x-y)+\cdots
$$

The form of this expression indicates that we should introduce the physical field $\mathcal{Y}^{(x, l, \alpha)}=$ $\left.\left(-\partial^{2}\right)^{1 / 2} \Phi^{(x, l, \alpha}\right)$.

The field strength for small $\{\Phi\}$ may be approximated as

$$
\mathcal{F}_{j k}^{\alpha} \approx \delta \mathcal{F}_{j k}^{\alpha}=\left[\epsilon^{k m l} \mathcal{D}_{j} \mathcal{D}_{m}-\epsilon^{j m l} \mathcal{D}_{k} \mathcal{D}_{m}+\mathcal{F}_{j k} \frac{1}{\mathcal{D}^{2}}(* \mathcal{F})_{l}\right]_{\alpha \beta} \delta \Phi^{(x, l, \beta)}
$$

The expansion of the potential energy in dual-Coulomb gauge is therefore

$$
\int \frac{1}{4}\left(\mathcal{F}_{j k}^{\alpha}\right)^{2}=\int \frac{1}{2} \Phi^{(x, l, \alpha)}\left(\partial^{2}\right)^{2} \Phi^{(x, l, \alpha)}=\int \frac{1}{2} \mathcal{Y}^{(x, l, \alpha)}\left(-\partial^{2}\right) \mathcal{Y}^{(x, l, \alpha)} .
$$

The effective Hamiltonian in $3+1$ dimensions is

$$
H_{\mathrm{eff}}=\int\left\{-\frac{e_{0}^{2}}{2} \frac{\delta^{2}}{\left(\delta \mathcal{Y}^{(x, l, \alpha)}\right)^{2}}+\frac{e^{2}}{2} \mathrm{~W}[\mathcal{Y}]+\frac{1}{2 e_{0}^{2}}\left(\partial_{j} \mathcal{Y}^{(x, l, \alpha)}\right)^{2}+\cdots\right\}
$$

where $\mathrm{W}$ is now

$$
\mathrm{W}[\mathcal{Y}]=(\operatorname{det} G)^{-\frac{1}{4}} \frac{\delta}{\delta \Phi^{(x, l, \alpha)}}\left[G^{(x, l, \alpha)(y, m, \beta)} \frac{\delta}{\delta \Phi^{(y, m, \beta)}}(\operatorname{det} G)^{\frac{1}{4}}\right] .
$$

The mass gap is obtained by expanding $\mathrm{W}$, as in $2+1$ dimensions.

Notice that the form of the effective Hamiltonian (10.13) will change if we impose a a different gauge condition on the dual gauge field. Furthermore the right-hand side of (10.14) is clearly dual-gauge dependent. This is how the dual gauge invariance is preserved.

\section{Conclusions and Outlook}

Through a non-Abelian decomposition formula for differential one-forms, we have found a special hypersurface in the space of connections. Gauge transformations are normal to this hypersurface, which makes it a useful tool in the geometry of gauge configurations. We have found expressions for the curvature tensor and sectional, Ricci and scalar curvatures of this hypersurface for $(\mathrm{D}+1)$-dimensional gauge theories. The hypersurface coordinate was explicitly determined in terms of the gauge connection in 2+1 dimensions. A Hamiltonian formalism close to that of Karabali, Kim and Nair

was worked out. Finally, we discussed an effective Hamiltonian of the dual degrees of freedom. 
There are many avenues which remain to be explored. Though our methods are evidently related to Karabali, Kim and Nair's $(2+1)$-dimensional formalism, the connection is not complete. We do not yet have a mapping between their degrees of freedom and ours. Furthermore, we do not yet see the connection between our ideas and Nair and Yelnikov's [13] in 3+1 dimensions. A mass for the dual gauge field in $3+1$ dimensions implies a type of dual Higgs phase. Though our duality is not the same as Kramers-Wannier duality, we believe that this would imply that the vacuum is a chromoelectric superconductor [19]. We hope that this issue will become clearer as we understand dual gauge invariance more completely.

The most urgent project is to regularize the determinant of the metric (in $2+1$ and $3+1$ dimensions) in order to obtain the dual Hamiltonian more explicitly. In this way, the mass gap and perhaps much of the physics can be understood, at least at strong coupling.

The geometry of the hypersurface needs to be examined more closely. We have discussed how to compute the Ricci tensor and curvature scalar in dimensional regularization, but the details of this computation are not finished.

Though we have mentioned the use of dimensional regularization in Section 6, we have not extensively discussed cut-off methods in this paper. A simple gauge-invariant regulator is to drop high-momentum (short-wavelength) components from $\{\Phi\}$. There is no obvious inconsistency with this idea in $2+1$ dimensions. In $3+1$ dimensions, such a procedure may explicitly break dual gauge invariance; perhaps this is not a serious difficulty, and it is enough to have ordinary gauge invariance. We have already explored a lattice version of our method, where all gauge invariances are preserved. In a Hamiltonian lattice formalism, where the gauge group is enlarged to $\operatorname{GL}(N, \mathbb{C})$, then broken to $\mathrm{SU}(N)$, a linear transformation $L$ can be defined. Another lattice formulation, with approximate conformal invariance of the kinetic term in $2+1$ dimensions, has been presented in a new paper by Rajeev [20]. It should be quite interesting to see whether an analogue of $L$ exists in his approach.

\section{Acknowledgements}

We thank both D. Karabali and V.P. Nair for discussions concerning the relation between the work presented here and that in references [9, 10, 13, and for comments on the manuscript.

\section{References}

[1] V.N. Gribov, Nucl. Phys. B139 (1978) 1; M.S. Narasimhan and T.R. Ramadas, Commun. Math. Phys. 67 (1979) 121; O. Babelon and C.M. Viallet, Phys. Lett. 101B (1981) 45; M. Asorey and P.K. Mitter, Commun. Math. Phys. 80 (1981); P. van Baal, International Workshop on Nonperturbative issues in QCD, Trento, Italy, D. Diakonov ed. (1995), hep-th/9511119 
[2] I.M. Singer, Commun. Math. Phys. 60 (1978) 7; Physica Scripta 24 (1981) 817.

[3] O. Babelon and C.M Viallet, Commun. Math. Phys. 81 (1981) 515.

[4] P. Orland, hep-th/9607134 (1996).

[5] M. Kudinov, E. Moreno and P. Orland, Zakopane 1997: New Developments in Quantum Field Theory (1997) 315, P. Damgaard and J. Jurkiewicz ed., Plenum Press, New York (1998).

[6] P. Orland and G.W. Semenoff, Nucl. Phys. B576 (2000) 627.

[7] M.B. Halpern, Phys. Rev. D16 (1977) 1798; J. Goldstone and R. Jackiw, Phys. Lett. B74 (1978) 81; B. Sakita, Phys. Rev. D21 (1980) 1067; A. Jevicki, BROWNHET-441, Nov. 1980, 13 pages; A.A. Migdal, Phys. Lett. B96 (1980) 333.

[8] J. Maldacena, TASI 2003 lectures on AdS/CFT, hep-th/0309246 and references therein.

[9] D. Karabali and V.P. Nair, Nucl. Phys. B464 (1996) 135; Phys. Lett. B379 (1996) 141.

[10] D. Karabali, C. Kim and V.P. Nair B524 (1998) 661; Phys. Lett. B434 (1998) 103; Nucl. Phys. B566 (2000) 331, Phys. Rev. D64 (2000) 025011.

[11] G. Dell'Antonio and D. Zwanziger, Nucl. Phys. B326 (1989) 333; Commun. Math. Phys. 138 (1991) 291.

[12] R.P. Feynman, Nucl. Phys. B188 (1981) 479.

[13] V.P. Nair and A. Yelnikov, hep-th/0302176.

[14] In two space dimensions, $L$ maps scalar Lie-algebra valued functions to vector Liealgebra valued functions. Hence it has, strictly speaking, no self-adjoint definition. However, fixing $j$ in $\mathrm{i} L_{(y, \beta)}^{(x, j, \alpha)}$ defines operators which should have self-adjoint extensions. The same idea should work for other numbers of space dimensions.

[15] E.B. Davies, Heat Kernels and Spectral Theory, Cambridge University Press (1989); I. Chavel, Eigenvalues in Riemannian Geometry, Academic Press, NY (1984).

[16] K. Gawedzki, Phys. Rev. D26 (1982) 3593.

[17] B. Simon, Functional Integration and Quantum Physics, Academic Press, NY (1979).

[18] Formally such solutions can always be obtained by integrating these equations for $b$ and $\bar{b}$. These solutions will not be normalizable and single-valued, in general, hence they are irrelevant. 
[19] S. Mandelstam, Bull. Am. Phys. Soc. 22 (1977) 541; G. 't Hooft, Nucl. Phys. B138 (1978) 1; S. Mandelstam, Phys. Rev. D19 (1979) 2391; G. 't Hooft, Nucl. Phys. B190 455 (1981).

[20] S.G. Rajeev, hep-th/0401202. 